\title{
Osteoarthritis Current Understanding
}

\author{
Abdul Ahad Khan* \\ Amina Hospital, Ajman, UAE \\ *Corresponding author: Abdul Ahad Khan, Amina Hospital, Ajman, UAE
}

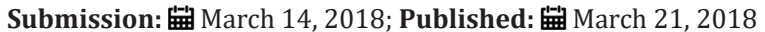

\section{Opinion}

Osteoarthritis has long been considered a 'wear and tear' disease leading to loss of cartilage. Progress in molecular biology in the 1990s has profoundly modified this paradigm. The discovery that many soluble mediators such as cytokines or prostaglandins can increase the production of matrix metalloproteinases by chondrocytes led to the first steps of an "inflammatory" theory. Recent experimental data have shown that subchondral bone may have a substantial role in the OA process, as a mechanical damper, as well as a source of inflammatory mediators implicated in the $\mathrm{OA}$ pain process and in the degradation of deep layer of cartilage.

Modern concepts of OA have been forever changed by modern imaging phenotypes demonstrating complex and multi-tissue pathologies involving cartilage, subchondral bone and increasingly recognized inflammation of the synovium. The synovium may show significant changes, even before visible cartilage degeneration has occurred, with infiltration of mononuclear cells, thickening of the synovial lining layer and production of inflammatory cytokines.

Complement is regulated by several catabolic and inflammatory mediators playing a key role in OA. It seems to be involved in many processes observed during OA development and progression, such as, extracellular cartilage matrix (ECM) degradation, chondrocyte and synoviocyte inflammatory responses, cell lysis, synovitis bone remodeling, osteophyte formation, and stem cell recruitment, as well as cartilage angiogenesis. Accumulating evidence demonstrates that complement activation is involved in the pathogenesis of $\mathrm{OA}$. Endogenous molecular products derived from cellular stress and extracellular matrix disruption can function as DAMPs to induce inflammatory responses and pro-catabolic events in vitro and promote synovitis and cartilage degradation in vivo via PRRs.

Innate immunity, a key player, in damage to the structure of the joint and the mechanism by which the host attempts to repair OA, affects all pathological stages of the disease. The disabling and painful disease $\mathrm{OA}$ is the most common form of arthritis. Strong evidence suggests that a subpopulation of OA patients has a form of OA driven by inflammation. Preliminary evidence from small clinical studies suggests that this subpopulation may benefit from anti-inflammatory treatment currently reserved for other inflammatory arthritides.

Management of osteoarthritis has been improved significantly over the last years with further improvement of the current therapeutic modalities, mainly total joint replacement. In parallel, a new era of biologic joint reconstruction has been initiated with use of biological tissue that aims to replace degenerative tissue.

\section{Kinematically aligned total knee replacement}

The main advantage of kinematically aligned total knee knee arthroplasty (TKA) is that allows proper position of TKA implants in order to restore the axis of both patellofemoral and tibiofemoral flexion/extension axes. As a consequence, it is independent of the femoral head and ankle position that outline the mechanical axis of the knee that is used as a reference of in mechanically aligned TKA. A randomized control trial confirmed these advantages by demonstrating the superiority of the kinematically aligned TKA compared to mechanical aligned TKA in terms of almost all clinical outcomes, such as Oxford Knee and Western Ontario and McMaster Universities Osteoarthritis Index (WOMAC) score, as well as better flexion and reduced pain. Also, a study with mean follow up of 6 years demonstrated implant survivorship of $97.5 \%$ with an overall Oxford Knee score of 42.7 As always, time will be the judge of whether the principles of kinematically aligned arthroplasty can be expanded and embraced in a way that they will improve outcomes and orthopedic practice.

\section{Computer assisted arthroplasty}

However, almost ten years after the first promising reports, computer assisted arthroplasty still struggles to prove its efficiency and establish its role in joint arthroplasty. This is due to the fact that there is not adequate support that the observed difference in alignment can be indeed translated into better subjective and outcome analysis scores. Robotic surgery is probably the application that can better depict the future involvement of technology in clinical orthopaedic practice. Even though robotic-assisted surgery started its first steps more than 20 years ago, with orthopaedics being one of the first specialities that used a robotic system, it was 
only in the last 5 yrs that its used has been remarkably extended.

\section{Biological joint reconstruction}

The concept of biological joint reconstruction envisions the replacement of the degenerative articular cartilage with biologic, non artificial, material that can be fully incorporated and integrated with the remaining healthy tissue. Even though the idea is miraculous, unfortunately, the regeneration of articular cartilage has not been a simple task so far. Primary chondrocytes remain the golden standard as well as a cell source for treatment of cartilage lesions, 20 years after their description of autologous chondrocyte implantation (ACI) that has initiated the concept of biological joint repair Despite all progress in the management of $\mathrm{OA}$, diet modification, weight management and supervised exercise program remained unchallenged.
Creative Commons Attribution 4.0

International License

For possible submissions Click Here

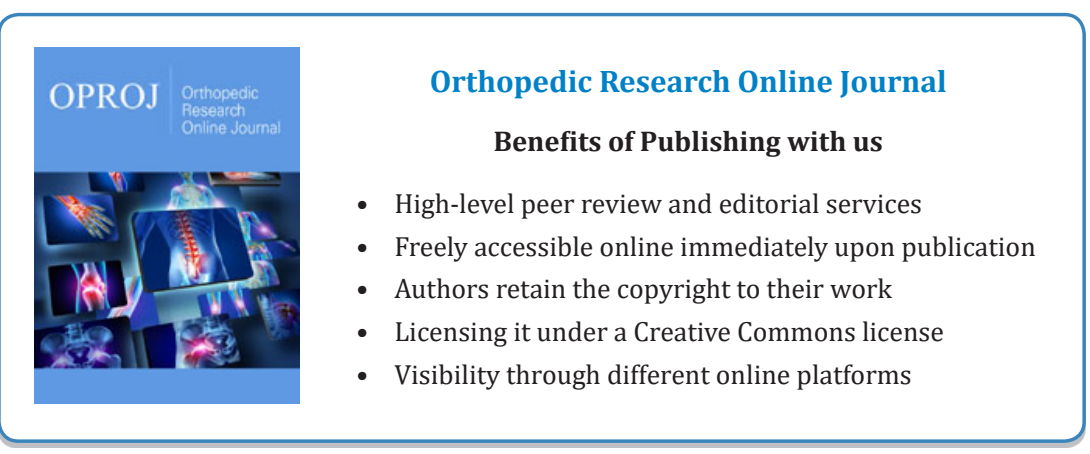

